\title{
ACCELERATION, CONTAINMENT AND EMISSION OF HIGH \\ ENERGY FLARE PARTICLES: RADIO EVIDENCE
}

\author{
A. BOISCHOT
}

Observatoire de Meudon, France

\begin{abstract}
The existence of non thermal radio bursts provide evidences for the acceleration of electrons in the solar atmosphere.

It is shown, from the characteristics of the bursts, that the electrons are accelerated in at least four different phases:

(1) An impulsive phase which gives $\mu \mathrm{ib}$ and III bursts.

(2) A gradual phase which gives $\mu$ IV and $S_{1}$ IV bursts.

(3) A quasi-continuous phase which gives $\mathrm{S}_{2} \mathrm{IV}$ bursts and noise storms.

(4) An acceleration by shock waves gives type II bursts.

(5) Eventually, another shock-wave acceleration giving the moIV burst.
\end{abstract}

\section{Introduction}

The large intensity of most of the radiobursts (brightness temperature between $10^{7}$ and $10^{14} \mathrm{~K}$ ) reveals that they are due to non-thermal mechanisms, i.e., that they take their energy from the kinetic energy of non-thermal particles.

In the following we shall assume that these particles are only electrons.

Most of the radiobursts are associated with some flare activity observed at chromospheric levels. We can divide them into two classes, somewhat arbitrarily: the bursts which are 'directly' related to the flare, begin at the same time or within a few minutes of the beginning of the $\mathrm{H} \alpha$ flare, and those the origin of which is also probably due to the flare activity, but which are observed after a longer delay, or are only statistically related to flares.

In the first group are the microwave impulsive burst ( $\mu \mathrm{ib})$ the type III and type $\mathrm{V}$ bursts, the type II and the type IV bursts the latter being made of different components that we shall discuss later on.

In the second group, we place the many types of bursts and emissions which form the noise storm activity: type I bursts on metric wavelengths, storm type III in decametric and longer waves, storm continuums, etc....

We shall not discuss the details of the characteristics of these bursts, which may be found in Kundu (1965), Wild et al. (1963), Wild and Smerd (1972), or Krüger (1972), but shall insist only on a few points which are important for our study of the high energy electrons; some other characteristics are given in Table $I$.

The observation of the radiobursts gives us the intensity vs time and frequency, the state of polarization and some informations on the source of emission: apparent size, position, motion, etc.... From that, we wish to determine as many characteristics of the energetic electrons as possible, in particular their total number and energy distribution, the pitch angle distribution, the duration of the acceleration process, the storage and release of the electrons, etc.... 


\section{TABLE I}

\begin{tabular}{|c|c|c|c|c|}
\hline Burst & $\begin{array}{l}T_{b} \\
(\max ) \\
{ }^{\circ} \mathrm{K}\end{array}$ & Duration & Source & Mechanism \\
\hline$\mu \mathrm{ib}$ & $10^{9}$ & 1 to $60 \mathrm{mn}$ & $\begin{array}{l}\text { fixed. Low corona } \\
\text { Double with opposite polarizations }\end{array}$ & $\begin{array}{l}\text { gyroemission } \\
B \sim 500 \mathrm{G}\end{array}$ \\
\hline$\mu \mathrm{IV}$ & $10^{9}$ & $10 \mathrm{mn}$ to $2 \mathrm{~h}$ & $\begin{array}{l}\text { fixed. Low corona } \\
\text { Double with opposite polarizations }\end{array}$ & $\begin{array}{l}\text { gyro- and } \\
\text { synchrotron }\end{array}$ \\
\hline$S_{1} I V$ & $?$ & $\begin{array}{l}10 \mathrm{mn} \text { to } 1 \mathrm{~h} \\
(?)\end{array}$ & $\begin{array}{l}\text { fixed } \\
0.1 \text { to } 1 R_{\odot}\end{array}$ & $\begin{array}{l}\text { synchrotron } \\
\text { (amplification?) }\end{array}$ \\
\hline moIV & $10^{12}$ & $10 \mathrm{mn}$ to $1 \mathrm{~h}$ & $\begin{array}{l}\text { moves upward to several } R_{\odot} \\
v \sim 100-1000 \mathrm{~km} \mathrm{~s}^{-1}\end{array}$ & synchrotron \\
\hline$S_{2} I V$ & $10^{12}$ & several hours & $\begin{array}{l}\text { fixed } \\
0.1 \text { to } 1 R_{\odot}\end{array}$ & $?$ \\
\hline III & $10^{12}$ & $\begin{array}{l}0.2 \mathrm{~s}(\mathrm{dm} \lambda) \text { to } \\
1 \mathrm{~h}(\mathrm{~km} \lambda)\end{array}$ & $\begin{array}{l}\text { at height of plasma resonance } \\
\text { Exciter: } v \sim 0.3 c\end{array}$ & $\begin{array}{l}\text { plasma oscillations } \\
\text { Electron stream }\end{array}$ \\
\hline V & $?$ & 1 to $10 \mathrm{mn}$ & $?$ & $?$ \\
\hline II & $10^{12}$ & 10 to $30 \mathrm{mn}$ & $\begin{array}{l}\text { at height of plasma resonance } \\
\text { Exciter: } v \sim 1000 \mathrm{~km} \mathrm{~s}^{-1}\end{array}$ & $\begin{array}{l}\text { plasma oscillations } \\
\text { Shock waves }\end{array}$ \\
\hline $\begin{array}{l}\text { Noise storm } \\
\text { continuum } \\
\text { m type I } \\
\text { dam type III }\end{array}$ & $\begin{array}{l}10^{10} \\
10^{12} \\
10^{12}\end{array}$ & $\begin{array}{l}\text { hours } \\
0.5 \mathrm{~s} \\
\text { few seconds }\end{array}$ & $\begin{array}{l}\text { fixed at a given frequency } \\
\text { fixed at a given frequency } \\
\text { Higher the source, lower the frequency }\end{array}$ & $\begin{array}{l}? \\
? \\
\text { plasma oscillations } \\
\text { Electron stream }\end{array}$ \\
\hline
\end{tabular}

This can be done, even partially, only if we know the mechanisms by which the radio-waves are excited. These mechanisms are often poorly understood, and quantitative studies are generally impossible.

Moreover, there is a limitation in the determination of the electron characteristics from the observation of radiobursts: propagation effects (refraction, cut-off, scattering, etc....) which are very difficult to take into account, can modify in a very sensitive manner the apparent intensity of the bursts and the characteristics of the sources, and then increase the difficulty of deriving the conditions within the sources from the observation of radiowaves at $1 \mathrm{AU}$. In particular, high energy electrons can exist in the solar atmosphere, which do not give any detectable radio emission.

\section{Identification of the Bursts}

$\mu \mathrm{ib}$ and type III are very clearly defined (Kundu, 1965). The problem of $\mu \mathrm{IV}$ is more complex. $\mu \mathrm{ib}$ and $\mu \mathrm{IV}$ are distinguished from the duration and shape of their time 


\begin{tabular}{|c|c|c|c|c|c|}
\hline \multicolumn{2}{|l|}{ Electrons } & \multicolumn{4}{|c|}{ Associated events } \\
\hline Number & Energy & $\begin{array}{l}\text { Hard } \\
\text { X-ray }\end{array}$ & $\begin{array}{l}\text { Electrons } \\
\text { (1 AU) }\end{array}$ & $\begin{array}{l}\text { Protons } \\
\text { (1 AU) }\end{array}$ & Flare \\
\hline $10^{30}-10^{32}$ & $10-100 \mathrm{keV}$ & + & + & - & small or large \\
\hline $10^{31}-10^{33}$ & $\begin{array}{l}10 \mathrm{keV} \text { to } \\
5 \mathrm{MeV}\end{array}$ & - & + & + & large \\
\hline $10^{32}-10^{33}$ & $\begin{array}{l}100 \mathrm{keV} \text { to } \\
5 \mathrm{MeV}\end{array}$ & - & + & + & large \\
\hline$?$ & $\begin{array}{l}100 \mathrm{keV} \text { to } \\
5 \mathrm{MeV} \text { (?) }\end{array}$ & - & $?$ & $?$ & large \\
\hline$?$ & $?$ & - & - & - & large \\
\hline $10^{32}-10^{35}$ & $10-100 \mathrm{keV}$ & + & + & - & small or large \\
\hline$?$ & $?$ & - & - & - & small or large \\
\hline$?$ & $?$ & - & - & - & large \\
\hline$?$ & $?$ & - & - & - & small or no flare \\
\hline$?$ & ? & - & - & - & \\
\hline $10^{32}-10^{35}$ & $10-100 \mathrm{keV}$ & - & - & - & \\
\hline
\end{tabular}

profile. They can be explained by electrons of similar characteristics, have the same brightness temperatures, similar spectra, similar intensity over duration ratios, etc.... This leads several authors to the conclusion that $\mu \mathrm{IV}$ is more likely the juxtaposition of several $\mu \mathrm{ib}$ (Takakura, 1967).

However, there is an important difference between the two types of bursts in their association with hard X-ray bursts.

The $\mu \mathrm{ib}$ is always associated with an impulsive hard X-ray burst, the two emissions being coincident in time and having the same duration (Peterson $e$ al., 1973). On the other hand, the long during $\mu \mathrm{IV}$ is accompanied only by a much shorter impulsive hard X-ray burst, similar to the above one, at the beginning of the radio emission.

It is then likely that the microwave emission in large flares is the superposition of a $\mu \mathrm{ib}$ and a longer and irregular emission corresponding to the $\mu \mathrm{IV}$. Then this requires two different processes for accelerating and/or trapping the high energy electrons.

There are even more discussions about the identification of the different compo- 
nents of the type IV event, and we cannot here discuss all the arguments presented by the different parties. In the author's opinion, three components can be distinguished.

The first component groups the $\mu \mathrm{IV}$ and the 'first stationary source' $S_{1} I V$. The latter is made of the dmIV (for a discussion of this dmIV see Wild et al., 1963) and the mIVA (Pick, 1961), or the mIVA $_{1}$ of Akinyan et al. (1971). The two have in common to come from fixed sources in the low corona, and to begin very shortly (a few minutes at most) after the flash phase of the flare.

The second component is the moving type IV, moIV (mIVB of Pick, 1961, or mIVA 2 of Akinyan et al., 1971) which is impossible to separate from the above $S_{1} I V$ and from the third component with only single frequency or spectral observations, but which is very clearly defined with position observations. Different structures have been recognized in the moIV bursts (Wild and Smerd, 1972, and references herein).

The third component, 'second stationary source' or $S_{2} I V$ corresponds to the mIVB (Wild et al., 1963) and possibly a part of the dmIV. It is observed on metric and longer wavelengths from a fixed source located low in the corona and close to the flare region. (It is not clear whether the source of $S_{1} I V$ is different from the source of $S_{2} I V$ or not.) This component is distinguished from $S_{1} I V$ by its polarization characteristics and its duration but, as it overlaps with $\mathrm{S}_{1} \mathrm{IV}$ and moIV, these three components cannot be separated on single frequency records or dynamic spectra.

Among the different characteristics of the bursts directly associated with flares, a very important one for our problem is their probability of occurrence with a given flare. Very good statistics are still lacking, but it has been shown that:

- $\mu \mathrm{ib}$ and III are observed with flares of any importance, and their intensity is not related to the importance of the flare.

- type IV bursts are only observed with large flares. Then they are accompanied by $\mu \mathrm{ib}$ and some type III bursts in the early phase of the event.

- for small flares, the relation between $\mu \mathrm{ib}$ and III is not clear. A flare can give either a $\mu \mathrm{ib}$ only, or some III only, or both types. But type III bursts are more frequent that $\mu \mathrm{ib}$.

- many flares, even of large importance, are not accompanied by any radioemission.

The location of the sources of emission is also very important to know; better observations are needed, specially on high frequencies.

$\mu \mathrm{ib}$ and $\mu \mathrm{IV}$ come from small regions very close to the flaring site, and more precisely - in a few observations - from small sources located above photospheric regions of different magnetic polarities, giving radiowaves of opposite circular polarizations. The source is fixed, within the accuracy of the determination. This accuracy is not good enough to show the variation of position with frequency, if any. It is not possible from the observation to distinguish between the sources of $\mu \mathrm{ib}$ and of $\mu \mathrm{IV}$.

$\mathrm{S}_{1}$ IV bursts also come from a region associated with the $\mathrm{H} \alpha$ source, but more extended. The observation of the emission up to long metric wavelengths (if not in the decameter range) means that the source extends at least to 0.5 or $1.0 R_{\odot}$ above the solar surface; microwave emission is no more observed from this upper part of the 
source. The average position of the source seems to change with frequency (lower the frequency, higher the source). This can be due to the effect of the coronal plasma on the low frequency limit of the gyroemission (Razin effect), to the variation of the energy distribution of the electrons and of the magnetic field inside the source, and also to the mechanism of emission.

For moIV burst, all the frequencies come from the same source, simple or complex (Boischot and Clavelier, 1968; Smerd and Dulk, 1971) which moves upward in the corona with a velocity of several hundreds of kilometers per second.

The source of the type III burst, on the contrary, varies with frequency, according to the plasma hypothesis, but the exact path of the source, relative to the position of the flare and to the coronal structures is not yet known exactly (Leblanc et al., 1974).

A last remark about the interpretation of the bursts on microwaves. It has been often emphasized that $\mu \mathrm{ib}$ and $\mu \mathrm{IV}$ show a variety of spectral shapes. It is very likely that this reflects, rather than some differences in the characteristics of the electrons and/or in the mechanisms of emission, a difference in the coronal plasma in and around the source. It is well known than in the low corona the optical depth for freefree absorption is large and, due to large density gradients, very sensitive to the frequency. Then the free-free absorption of the radio waves, which depends on the structure of the source (height and size) and of the surrounding plasma can vary with frequency in a different manner for different bursts (Ramaty and Petrosian, 1972).

\section{3. $\mu$ ib and Type III Bursts}

We shall first consider these two kinds of bursts together, for we can see, from Table I, that their emission can be explained by electrons of similar energies, between $10 \mathrm{keV}$ and $100 \mathrm{keV}$ and that they are observed at the same time.

The $\mu \mathrm{ib}$ is explained by the gyroemission of the electrons in a $\sim 500$ to $1000 \mathrm{G}$ magnetic field, and the type III by plasma oscillations excited by an ejected stream of such electrons.

The problem is to know if there is only one or two different acceleration processes to give the two kinds of bursts. It has been said sometimes that two accelerations are necessary because their duration must be different for the two bursts.

For $\mu \mathrm{ib}$, the life time of the electrons of energy $E$ radiating a gyroemission in a field of $1000 \mathrm{G}$, and a thermal density of $n$ electrons per cubic centimeter, is shown to be limited by collisions on thermal electrons.

$$
\tau_{c} \sim 2.2 \times 10^{9} \frac{E^{3 / 2}}{n \mathrm{Ln}},
$$

where $\mathrm{Ln}$ is the Coulomb logarithm $\sim 20$.

For a density $\sim 10^{10} \mathrm{~cm}^{-3}$ which has been claimed necessary to explain the hard X-ray bursts, we find $\tau_{c}=0.25 \mathrm{~s}$ and $7.5 \mathrm{~s}$ for $10 \mathrm{keV}$ and $100 \mathrm{keV}$ electrons respectively (de Feiter, 1972).

Then the observed duration of the $\mu \mathrm{ib}$, several minutes, requires that there must be 
a continuous acceleration of electrons during this time. This conclusion has been discussed (Takakura, 1972). It depends very much on the assumed density in the source, which is not known. If we take $n=10^{8} \mathrm{~cm}^{-3}$, which is not unlikely, we can reconcile the life time of the electrons with the duration of the bursts. However, we must note that the coincidence between the positions of the sources of $\mu \mathrm{ib}$ and that of the source of the slowly varying component (coronal condensations) and the increased probability to observe $\mu \mathrm{ib}$ when the slowly varying component is intense (Kundu, 1959) point to large values of the density in the source.

The duration of the acceleration process can neither be determined accurately from the characteristics of the type III bursts. It has often been said that the high frequency duration of a type III burst, 0.2 to $0.5 \mathrm{~s}$, gives a limit of the duration of the acceleration process, which has to be repeated to give the several type III bursts observed in a group. But we cannot disregard the possibility that a type III burst corresponds only to the head of an electron stream, or to a change of density in a quasicontinuous stream, and that the acceleration is more or less continuous during the duration of the entire group of type III, i.e. between $10 \mathrm{~s}$ and a few minutes.

Then there is nothing against assuming that $\mu \mathrm{ib}$ and type III are due to electrons accelerated simultaneously (Kane, 1972). In this line, the difference between the two kinds of bursts is to be explained by the conditions of trapping and release of these electrons.

For $\mu \mathrm{ib}$ the electrons are certainly trapped in the low corona over active centers. The double source structure, with different polarities, and the high magnetic field required for the emission mean that the trapping region has closed lines of force and is located above bipolar regions of strong magnetic fields, i.e., directly above the active regions.

On the contrary, the type III electrons must escape along an open line of force as indicated by the extension of the emission to very low frequencies. It has been said that the constancy of the exciter velocity derived from the frequency drift rate means that the electrons have a small pitch angle (the velocity parallel to $\mathbf{B}$ would increase with decreasing $B$ for electrons of large pitch angles). But this also can be discussed as it is now thought that the observed frequency drift corresponds to the velocity of the front of the stream, i.e. of the fastest electrons, and not to the average velocity of the stream.

The theory of type $\mathrm{V}$ burst is not yet clear, but it is possible that this burst is the emission of some of the electrons of the stream, scattered in the surrounding corona by density and magnetic field inhomogeneities.

In the above model the electrons, accelerated in the region of the $\mathrm{H} \alpha$ flare or close to it, must encounter either closed lines of force, to give $\mu \mathrm{ib}$, or open lines to give type III or both to give simultaneously the two kinds of bursts. For Sturrock (1972) this difference is due to the magnetic configuration associated with the flare.

An important result in this respect has been obtained recently by Axisa (1974). Studying the location of the flare (Flare Productive Sites) inside three active regions, he was able to show that there is a difference in the magnetic configurations near 
the FSP at the photospheric levels ( $\mathrm{H} \alpha$ magnetograms) between flares which are accompanied predominantly by either $\mu \mathrm{ib}$ or by type III. In the former case, the flare takes place along an inversion line $\left(B_{\|}=0\right)$ separating two compact magnetic regions of opposite polarities and large magnetic fields $(15<B<100 \mathrm{G})$; with this configuration, only closed magnetic lines of force are present around the flare site and most of the electrons remained trapped. Type III bursts are inhibited and a $\mu \mathrm{ib}$ observed (and also a hard X-ray burst).

When, on the contrary, on one side of the $B_{\|}=0$ line where the flare occurs there is an extensive region of weak magnetic fields, the particles can find open lines of force and the production of type III is favored.

When, in this last case the flares also affect a region of intense magnetic field such as the penumbral part of a spot, both type III and $\mu \mathrm{ib}$ are observed (and also a hard $\mathrm{X}$-ray burst).

Finally, one remark must be made about the escape of the type III electrons. The presence of a magnetic open structure is certainly not sufficient to explain the trajectory of the electrons. At the chromospheric level, many electrons would then leave the flare region on a line of force which turns down to the Sun at some altitude over the surface. These electrons would give emission of type $U$ bursts. But it is very remarkable that the proportion of type $U$ bursts to the normal type III is very small. This means that the electrons can 'recognize' even at the chromospheric level, the lines which are really open in the high corona.

This is possible probably because there is a change of density of the plasma related to the magnetic structure, which favors the propagation of the high energy electrons along or near these open lines (which are perhaps neutral lines). This requires that the roots of the magnetic structure lie very deep in the solar atmosphere, in the photospheric active region. This is an important conclusion for the structure and origin of coronal streamers, if we assume that the type III at large distance from the Sun propagate along such streamers.

This problem is also related to the propagation of the electrons in dense structures (Smith and Pneuman, 1972).

\section{4. $\mu I V$ and $S_{1} I V$ Bursts}

These bursts are received only at the time of large flares and last much longer than the $\mu \mathrm{ib}$ or the group of type III bursts. Moreover, they are very often accompanied by an emission of high energy protons and relativistic electrons observed in the interplanetary medium, unlikely to the $\mu \mathrm{ib}$ and type III, which correspond only to medium energy electron events, without protons. The acceleration process for $\mu \mathrm{IV}$ and $\mathrm{S}_{\mathbf{1}} \mathrm{IV}$ on the one hand, and for $\mu \mathrm{ib}$ and type III on the other must then be different.

It follows that the differences between the two classes of bursts pose a serious problem.

We have, for the flash phase, electrons of medium energy which can, in many cases, escape along open lines of force to give type III bursts. Later on, we get electrons of even larger energy, in at least the same number - if the determinations of Table I are 
correct - which are also observed in the interplanetary medium, but which do not give rise to type III bursts.

An explanation of this phenomenon can be found in the nature of the acceleration process. If, as indicated by space observations of electron and proton events, the energy spectrum of the accelerated particles follows approximately a power law in both cases, it has only negative slopes, and the electrons cannot excite any plasma instability and type III burst. This can be done only if a positive slope in the velocity distribution function is obtained due to the dispersion of the particles propagating with different velocities. But in turn this requires that the acceleration process and/or the liberation of the electrons are impulsive enough to give this dispersion. This is certainly the case for the flash phase acceleration. But the second acceleration, which gives the $\mu \mathrm{IV}$ and $\mathrm{S}_{1} \mathrm{IV}$ bursts, starts probably more gradually, so that the electrons are unable to excite type III bursts. This model is in agreement with some new observations of the energy distribution in the interplanetary space (Lin, 1972; Lin et al., 1973).

At the beginning of an electron event, the energy distribution function presents a maximum in the $100 \mathrm{keV}$ range. As time evolves, this maximum drifts toward lower and lower energies, up to the time when the true energy spectrum of the accelerated electrons, with only a negative slope, is observed. This spectrum is then observed as long as there is no rapid variation in the number of electrons accelerated.

If this stationary state, with only a negative slope, is attained before the number of electrons is large enough to excite plasma waves, type III bursts will not be observed.

The difference in impulsiveness of the acceleration process (or release) also explains the difference of the starting frequency of different type III bursts.

In this second phase, the electrons are accelerated up to energies higher than in the case of the flash phase, and are accompanied by high energy protons. They are first trapped in the same region that those giving the $\mu \mathrm{ib}$ i.e. in closed lines of force of intense magnetic fields over the active region where the flare is observed. Then, they diffuse more or less rapidly to higher regions of the corona where they radiate the $\mathrm{S}_{1}$ IV burst. This diffusion is more important than for the flash phase electrons (which do not give metric emission) because the energy of the electrons is larger and probably, because there is more turbulence in the trapping region due to the larger importance of the flare.

The problem of the distinction between $\mu \mathrm{IV}$ and $\mathrm{S}_{1} \mathrm{IV}$ has been discussed by Böhme (1972) who invokes a separate acceleration process for the latter. However, the minimum in the intensity often observed in the decimeter range can found another explanation:

The type IV on decimetric and metric wavelengths can attain very large intensities soon after the beginning of the emission (i.e. at times corresponding to the dmIV and mIVA components) with flux densities up to $10^{-18} \mathrm{~W} \mathrm{~m}^{-2} \mathrm{~Hz}^{-1}$ and a source size about $10^{\prime}$ (which is probably an overestimation). The brightness temperature of the source is then sometimes larger than $10^{12} \mathrm{~K}$ at, say, $\lambda=3 \mathrm{~m}$. This high brightness temperatures can be reached by an incoherent mechanism only if the electrons have an 
average energy of $80 \mathrm{MeV}$. This is certainly too large to fit the other observations.

Then we must conclude that the mechanism giving the decimeter and meter emission of type IV is, at least partially, coherent, or in other words, that the synchrotron waves are coherently emitted or amplified in the corona. This amplification is very likely a function of frequency and of the local conditions. Then it can explain at the same time:

- the minimum observed between $\mu \mathrm{IV}$ and the emission at longer wavelengths.

- the intensity observed in the metric range which is sometimes much larger than that of the $\mu \mathrm{IV}$ burst.

- the high variability often observed in the dmIV, which we have no reason to distinguish from the mIVA.

- the many structures of 'modulation' observed in the metric range (Slottje, 1972).

\section{5. $S_{2}$ IV Bursts and Noise Storms}

$\mathrm{S}_{2}$ IV bursts (i.e. mIVB and perhaps a part of the dmIV) are observed after large flares, and last often for several hours. Most of their characteristics are similar to those of the continuum of the noise storms, and sometimes we can observe a $S_{2} I V$ burst turning into a normal noise storm after a few hours. This is the reason why we shall study them together.

The mechanisms of the emission which are at the origin of $\mathrm{S}_{\mathbf{2}} \mathrm{IV}$ bursts and noise storm activity (continuum and fast bursts) are still unknown. Therefore we have no idea of the amount and the energy of the electrons required, and all our conclusions about the acceleration process will be very speculative. However, we can show that these electrons are certainly accelerated in a process different from the two previous ones.

An important difference between $S_{2} I V$ burst and noise storm on the one hand, and the bursts studied above on the other, is that the former are accompanied neither by microwave emissions, although they can be as intense as the $S_{1}$ IV burst in the metric range, nor by interplanetary electrons. (Increases of high energy electrons during the passage of large active centers on the disc have been observed but with an intensity much smaller than those accompanying the flash phase of the flare)(McDonald, 1970).

Another noteworthy point is the difference of nature between the metric and decametric noise storm bursts (Boischot et al., 1970, 1971). The former are essentially type I bursts, the mechanism of which being unknown, and the latter mainly type III bursts beginning at a frequency between 60 and $30 \mathrm{MHz}$, and extending down to much longer wavelengths. These type III bursts have the same average characteristics than the type III observed at the flash phase of a flare, except their starting frequency.

The high intensity of the metric continuum, and of type I bursts, requires certainly non thermal electrons in the source for many hours or days, i.e. in regions between 0.2 and $1.0 R_{\odot}$ (but not below to explain the absence of $\mathrm{cm}$ and $\mathrm{dm}$ emission).

The type III storm bursts reveal on another hand that high energy electrons - between 10 to $100 \mathrm{keV}$ if we believe the theory of type III bursts - escape from this 
source into the interplanetary medium. During an average noise storm as many as $10^{4}$ type III burst can be observed in a day. If, as assumed for the isolated type III, each burst requires $10^{32}$ to $10^{35}$ electrons, we arrive at $10^{36}$ to $10^{39}$ electrons of 10 to $100 \mathrm{keV}$ every day. This number is certainly too large to be attributed to one single or even a few flares, and requires a quasi-continuous acceleration during the whole life time of the storm.

The above figure is derived from the observation of type III bursts only, and is probably underestimated, as it has been shown that the so called type III $_{b}$ bursts (De la Noë and Boischot, 1972), nearly as frequent as the normal type III burst in the decameter range, must also be attributed to the escape of electrons of similar energy, and reveal that many other electrons can escape the storm region without giving any radio emission.

Then we arrive at the conclusion that the noise storm and the $S_{2} I V$ bursts require another source of acceleration quasi-continuous during the life time of the noise storm.

We have for the moment very little idea of what this process is, and where it occurs. It can take place in the active center at chromospheric or low coronal levels. However to explain in the absence of microwave emission, it is more likely that it takes place in current sheets or near neutral points higher in the corona, at an altitude of about $1 R_{\odot}$ (Steward and Labrum, 1972).

The relation between the noise storms and the direct observation of high energy electrons in the interplanetary space poses another problem. It seems from the published observations that the increase of the number of these electrons at the time of noise storms is much smaller, if it even exists (Anderson, 1969) than the increase after an isolated group of flare associated type III bursts. This requires an explanation which could be that the number and energy of the electrons claimed to be necessary to excite a type III burst are much overestimated, and that smaller fluxes can as well give storm type III bursts, with the same average characteristics. This, at the same time, would help solving the problem of the total number of electrons escaping during a noise storm, discussed above.

If true, this conclusion would be very important for the general theory of the type III burst.

\section{Moving Type IV Bursts (moIV)}

The acceleration process leading to the emission of the moIV is not known. Two possibilities exist, both as likely.

The radiating electrons could be some of the electrons accelerated in the second phase (which gives $\mu \mathrm{IV}$ and $\mathrm{S}_{1}$ IV bursts) which remained trapped in a special magnetic configuration (plasmoï) ejected from the flaring region in the high corona (Schmahl, 1972) or which propagate along open lines of force and encounter some local reinforcements of magnetic field such as may be observed in a solitary wave in parallel propagation (Warwick, 1969).

- The electrons can be either accelerated locally in the wake of a shock wave 
moving up in the corona. Such an acceleration process has been studied by Lacombe and Mangeney (1969) and more recently by Mangeney (1974).

Even though the problems of confinment of high energy electrons in a plasmoid, and of their acceleration by a shock wave is very interesting, we have too little information on the nature of the electrons, and too little place in this paper to discuss them in more details.

\section{Type II Bursts}

We shall be brief also about type II bursts. It is generally agreed that the electrons required to excite plasma waves are accelerated either in the front or in the wake of a shock wave. We have for the moment too few informations on these electrons (number, energy distribution,...) to be able to discuss the details of the acceleration process. The recent observation of type II bursts in the interplanetary medium down to $500 \mathrm{kHz}$ (Stone and Fainberg, 1972) means that the electrons are certainly accelerated by the shock wave and are not flare electrons trapped in or near the shock.

\section{Summary}

To sum up, we propose the following classification of the acceleration phenomena, based on the study of the radio bursts.

(1) Coincident with the flash phase of the flare, an 'impulsive' acceleration with a duration of a few seconds to a few minutes, giving electrons of medium energy (1 to 100 $\mathrm{keV})$ without protons. These electrons are either trapped near the flare center in a high magnetic field closed to the surface of the Sun to give the $\mu \mathrm{ib}$ burst (and the impulsive hard X-ray burst) or they escape along open lines of force to give the type III bursts. Their energy is not sufficient to allow their diffusion from the trapping region into the higher corona where they would have given metric emission, but some of the type III electrons might be scattered to give the type $\mathrm{V}$ burst.

(2) In the case of large flares only a second phase of acceleration more gradual and lasting longer (minutes to hours) giving electrons of higher energy (or a flatter spectrum) $(100 \mathrm{keV}$ to a few $\mathrm{MeV})$ and also protons. These electrons are first trapped in the same closed loops region which gives the $\mu \mathrm{ib}$, where they radiate the $\mu \mathrm{IV}$ burst; then they diffuse to larger heights to give the $S_{1} I V$ burst.

(3) A third phase which is enhanced just after large flares but works also in the absence of flare. We have no information on the number and energy of the accelerated electrons. They give the $S_{\mathbf{2}}$ IV bursts and the noise storms. This acceleration might take place near the flare itself, in the active center, but more likely at high altitude in the corona.

(4) Another kind of acceleration, in the wake of a shock wave will give the moIV, but a possibility remains that this burst is due to the electrons accelerated in phase 2 and trapped in special moving structures such as plasmoïds.

(5) Also in the front or in the wake of a shock wave, an acceleration process at the origin of the type II burst. 


\section{References}

Akinyan, S. T., Mogilevsky, E. I., Bohme, A., and Krüger, A.: 1971, Solar Phys. $20,112$.

Anderson, K. A.: 1969, Solar Phys. 6, 111.

Axisa, F.: 1974, Solar Phys. 35, 207.

Bohme, A.: 1972, Solar Phys. 25, 478.

Boischot, A. and Clavelier, B.: 1968, Ann. Astrophys. 31, 445.

Boischot, A., De la Noë, J., Du Chaffaut, M., and Rosolen, C. : 1971, Compt. Rend. Acad. Sci. $272,166$.

Boischot, A., De la Noë, J., and Pedersen, B. M.: 1970, Astron. Astrophys. 4, 159.

De Feiter, L. D.: 1972, Space Sci. Rev. 13, 827.

De la Noë, J. and Boischot, A.: 1972, Astron. Astrophys. 20, 55.

Kane, S. R.: 1972, Solar Phys. 27, 174.

Krüger, A.: 1972, Physics of Solar Continuum Radiobursts, Academic Verlag, Berlin.

Kundu, M. R.: 1959, Ann. Astrophys. 22, 1.

Kundu, M. R.: 1965, Solar Radio Astronomy, Interscience Publ., New York.

Lacombe, C. and Mangeney, A.: 1969, Astron. Astrophys. 1, 325.

Leblanc, Y., Kuiper, T. B. H., and Hansen, S.: 1974, this volume, p. 227.

Lin, R. P.: 1972, in R. Ramaty and R. G. Stone (eds.), Proc. of High Energy Phenomena on the Sun, GSFC Preprint X-693-73-193, p. 439.

Lin, R. P., Evans, L. G., and Fainberg, J.: 1973, Astrophys. Letters 14, 191.

Mangeney, A.: 1974, this volume, p. 387.

McDonald, F. B.: 1970, in Manno and Page (eds.), Intercorrelated Satellite Observations Related to Solar Events, D. Reidel Publ. Co., Dordrecht, Holland, p. 34.

Peterson, L. E., Datlowe, D. W., and McKenzie, D. L.: 1973, in R. Ramaty and R. G. Stone (eds.), Proc. of High Energy on the Sun, GSFC Preprint X-693-73-193, p. 132.

Pick, M.: 1961, Ann. Astrophys, 24, 183.

Ramaty, R. and Petrosian, D.: 1972, Astrophys. J. 178, 241.

Schmahl, E. J.: 1972, Proc. Astron. Soc. Australia 2, 95.

Slottje, C. : 1972, Solar Phys. 25, 210.

Smerd, S. F. and Dulk, G. A.: 1971, in R. Howard (ed.), 'Solar Magnetic Fields', IAU Symp. 49, 616.

Smith, D. F. and Pneuman, G. W.: 1972, Solar Phys. 25, 461.

Stewart, R. T. and Labrum, N. R.: 1972, Solar Phys. 27, 192.

Stone, R. G. and Fainberg, J.: 1973, in R. Ramaty and R. G. Stone (eds.), Proc. of High Energy Phenomena on the Sun, GSFC Preprint X-693-73-193, p. 519.

Sturrock, P. A.: 1972, Solar Phys. 23, 438.

Takakura, T.: 1967, Solar Phys. 1, 304.

Takakura, T.: 1972, Solar Phys. 26, 151.

Takakura, T.: 1973, in R. Ramaty and R. G. Stone (eds.), Proc. of High Energy Phenomena on the Sun, GSFC Preprint X-693-73-193, p. 179.

Warwick, J. W.: 1968, Solar Phys. 5, 111.

Wild, J. P. and Smerd, S. F.: 1972, Ann. Rev. Astron. Astrophys. 10, 169.

Wild, J. P., Smerd, S. F., and Weiss, A. A.: 1963, Ann. Rev. Astron. Astrophys. 1, 291.

\section{DISCUSSION}

Pick (on Boischot paper): I would like to comment on the old classification of type IV bursts and the new one. (a) What we called type IVMA occurred at the time of the type IV $\mu$. Its main characteristic was a similar behavior in the intensity evolution to that of the microwave burst. This phase very likely corresponds to the 'flare continuum' which is a stationary source as revealed by the Culgoora observations. The electron energy would be less than $1 \mathrm{MeV}$. (b) The type IVMB corresponds to the moving type IV burst as described by Boischot (Compt. Rend. Acad. Sci., 1959). Concerning the dm type IV burst, both moving and stationary type IV bursts are observed - the large fluctuations that you reported are in fact observed during the stationary part. The sources of the metric and decimetric type IV burst do not correspond to the same position; the intensity behaviors are not similar, large fluctuations being observed in the dm range.

Kai: Flare-continua generally coincide in time with microwave IV bursts. The example of a flarecontinuum source, which I mentioned before, suggests that microwave IV bursts originate in the most inner part of the extended flare-continuum source. 
Schmidt: I agree with Dr Sturrock that it is still somewhat premature to construct a good theoretical model for the phase I. But I think some points made by several speakers at this symposium point towards a phenomenological model which would take into account the effects of the low-lying current sheets along the legs of the typical helmet configuration. Dr Pneuman described these current sheets for the static configuration. Dr Boischot has mentioned these current sheets as possible source regions of type III bursts in phase I, whereas the type III bursts which come in phase III may come from the upper current sheet which is located above the two others. The time profile of the total energy release during a major flare may support this view. In phase I the major part of the flare energy is still confined in the region inside of the two lower current sheets, so that they are more compressed than usual (by dynamic effects) and that their rate of dissipation goes up. On the other hand, during phase III the major part of the flare energy has already left the whole flare region. As described in the model of Carmichael mentioned by Dr Sturrock, the upper current sheet will then reconnect the magnetic flux expanded earlier very effectively.

Pneuman: I would like to comment upon the very important point that Dr Boischot made regarding the commonness of type III bursts and the relative rarity of $U$-bursts which are supposed to have the same physical explanations. If this is true, then doesn't this argue strongly against a purely chromospheric origin of flares? If the flare occurred first in the chromosphere then one would expect electrons to be ejected in all directions - both along closed and open field lines. Why do the electrons always seem to find the open lines?

On the other hand, the explanations might not be the same. I believe that closed flux tubes contain more material than open field lines. Hence, the electrons that would normally give rise to $U$-bursts are quenched quenched due to coulomb collisions whereas the electrons on open field lines can get out of the lower corona more easily. 